\title{
SIRT5 promotes cell proliferation and invasion in hepatocellular carcinoma by targeting E2F1
}

\author{
LIANG CHANG $^{1,2}$, LIANG XI ${ }^{3}$, YUBIN LIU ${ }^{2}$, RUI LIU ${ }^{3}$, ZHONGSHI WU $^{2}$ and ZHIXIANG JIAN ${ }^{1,2}$ \\ ${ }^{1}$ Southern Medical University, Guangzhou, Guangdong 510515; ${ }^{2}$ Department of General Surgery, Guangdong General Hospital, \\ Guangdong Academy of Medical Sciences, Guangzhou, Guangdong 510000; ${ }^{3}$ Department of Surgical Oncology, \\ Inner Mongolia Baotou Steel General Hospital, Baotou, Inner Mongolia 404010, P.R. China
}

Received November 20, 2016; Accepted June 29, 2017

DOI: $10.3892 / \mathrm{mmr} .2017 .7875$

\begin{abstract}
Sirtuin 5 (SIRT5) is a member of the NAD ${ }^{+}$-dependent class III protein deacetylases. Although it is known that SIRT5 deacetylates and activates urate oxidase in the liver mitochondria of mice, the mechanism of SIRT5 in the proliferation of hepatocellular carcinoma (HCC) remains to be fully elucidated. The present study investigated the expression and functional significance of SIRT5 in HCC, and examined the relevant mechanism. SIRT5 was found to be upregulated in HCC tissues and cell lines, and the higher expression of SIRT5 indicated poorer overall survival. Reverse transcription-quantitative polymerase chain reaction analysis, western blot analysis, chromatin immunoprecipitation analysis, and luciferase reporter gene, proliferation and Transwell assays were performed to elucidate the function of SIRT5 in the regulation of cell proliferation and invasion in human HCC. Functionally, it was observed that the inhibition of SIRT5 significantly suppressed HCC cell proliferation and invasion, whereas the overexpression of SIRT5 promoted HCC cell proliferation and invasion in vitro. E2F transcription factor 1 (E2F1) was identified as a novel target gene of SIRT5. In addition, the knockdown of SIRT5 induced the expression of E2F1, and the knockdown of E2F1 in HCC cells partially reversed the effect of SIRT5 in promoting cell proliferation and invasion. Collectively, these data provide the first evidence, to the best of our knowledge, that the SIRT5 gene has an important regulatory role in liver carcinogenesis, and may function as a novel potential therapeutic target for HCC.
\end{abstract}

Correspondence to: Professor Zhixiang Jian, Department of General Surgery, Guangdong General Hospital, Guangdong Academy of Medical Sciences, 106 Zhongshan Er Road, Yuexiu, Guangzhou, Guangdong 510000, P.R. China

E-mail: zhixiang8877@sina.com

Key words: sirtuin 5, proliferation, invasion, hepatocellular carcinoma, E2F transcription factor 1

\section{Introduction}

Liver cancer is the fifth most prevalent diagnosed cancer and the second most common cause of cancer-associated mortality worldwide (1). Of all malignant types of liver cancer, hepatocellular carcinoma $(\mathrm{HCC})$ is the most common type in adults, which accounts for $70-85 \%$ of cases (2), and the highest incidence rates are reported to occur in Asia and Africa (3). HCC is an insidious disease without symptoms of pain, which results in diagnosis at a late stage (4). Although there has been significant progress in HCC therapy, recurrence and metastasis remain major obstacles in improving outcomes following surgical resection and liver transplantation $(5,6)$. The 5-year survival rate is limited to $30 \%$. Therefore, the investigation of novel effective biomarkers and targets to develop novel effective strategies is urgently required.

The sirtuins are a family of orthologues, which share extensive homologies with the silent information regulator 2 (Sir2) gene in yeast. In mammals, sirtuins comprise seven members, termed sirtuin (SIRT)1-SIRT7, respectively, and are important in the regulation of metabolism, cellular proliferation, aging, survival, and oncogenesis or tumor suppression in the progression of cancer (7-9).

Among the sirtuin family members, SIRT5 has been investigated the least, and the role of SIRT5 in carcinogenesis remains to be elucidated, which requires further detailed investigation. In the present study, the expression status of SIRT5 in HCC specimens and cell lines were evaluated, and the functional contribution of SIRT5 in the progression of HCC was investigated.

\section{Materials and methods}

Cell culture. The human HCC SMMC-7721, HepG2, LO2 and Huh7 cell lines, and HEK-293T cells were purchased from the American Type Culture Collection (Manassas, VA, USA). Non-tumorigenic immortalized liver cell line MiHA were purchased from the Cell bank of the Institute of Biochemistry and Cell Biology (Academy of Life Science; Shanghai, China). The cells were placed in Dulbecco's modified Eagle's medium (DMEM) (Invitrogen; Thermo Fisher Scientific, Inc., Waltham, MA, USA) containing $10 \%$ fetal bovine serum (FBS) (Invitrogen; Thermo Fisher Scientific, Inc.), penicillin (100 U/ml) and streptomycin $(100 \mu \mathrm{g} / \mathrm{ml})$ (Invitrogen; Thermo 
Fisher Scientific, Inc.), and were cultured in a $5 \% \mathrm{CO}_{2}$ incubator at $37^{\circ} \mathrm{C}$.

Reagents and transfection. The relative small interfering (si)RNAs and the negative control siRNA were purchased from Santa Cruz Biotechnology, Inc. (Dallas, TX, USA). The siRNA sequences were as follows: SIRT5 siRNA\#1, 5'-CCGGGCGTGCCAGTGGCTGAATTTACTCGAGTAA ATTCAGCCACTGGCACGCTTTTTG-3'; SIRT5 siRNA\#2, 5'-CCGGCTGAGTACTGAACAATCTAAACTCGAGTTTA GATTGTTCAGTACTCAGTTTTTG-3'; E2F1 siRNA\#1, 5'-CCGGCCCAACTACAAGCTGTGGATTCTCGAGAATC CACAGCTTGTAGTTGGGTTTTTG-3'; E2F1 siRNA\#2 (5'-CCGGGCCAAGAAGTCCAAGAATCATCTCGAGATGA TTCTTGGACTTCTTGGCTTTTTG-3'); and non-silencing siRNA 5'-CTAGCCCGGTTCTCCGAACGTGTCACGTAT CTCGAGATACGTGACACGTTCGGAGAATTTTTTTAAT-3'. The cells $\left(2 \times 10^{5}\right)$ were seeded in 6 -well plates overnight and transfected with $5 \mu \mathrm{M}$ siRNAs using Lipofectamine ${ }^{\circledR}$ RNAiMAX (Invitrogen Life Technologies; Thermo Fisher Scientific, Inc.). After 1-2 days, the transfected cells were harvested for RNA or protein extraction. The antibodies used in the present study were as follows: Anti-SIRT5 antibody (cat. no. ab105040), anti-E2F transcription factor 1 (E2F1) antibody (cat. no. ab179445), and anti- $\beta$-actin antibody (cat. no. ab8226; all from Abcam, Cambridge, UK).

Generation of lentivirus. HEK-293T cells were used to produce the lentivirus. The HEK-293T cells $\left(2 \times 10^{7}\right)$ were grown to $50-60 \%$ confluence in $15 \mathrm{~cm}$ dishes, and the purified shRNA plasmids and the lentivirus GenePharm (Shanghai, China) were co-transfected into the HEK-293T cells using Lipofectamine 2000 reagent (Invitrogen Life Technologies; Thermo Fisher Scientific, Inc.), according to the manufacturer's protocol. After $6 \mathrm{~h}$, the medium was removed and fresh medium was added. At 4 days-post transfection, the media were harvested. The viruses were released by three freeze/thaw cycles and stored at $-80^{\circ} \mathrm{C}$ until use. For viral infection, $30 \mu \mathrm{l}$ of viral stock solution was added to the culture medium in each group. The expression of SIRT5 was determined in the infected cells using reverse transcription-quantitative polymerase chain reaction (RT-qPCR) analysis.

Patients and tissue specimens. A total of 55 fresh HCC tissue samples and paired non-cancerous lung tissue samples were obtained during surgery in the first surgical procedure following the diagnosis of HCC between January 2005 and December 2009 at the Department of Pathology at Guangdong General Hospital (Guangzhou, China). None of the patients had received radiotherapy or chemotherapy prior to surgery. Tumor-node-metastasis (TNM) classification was defined according to the American Joint Committee on International Union Against Cancer (10). The stage IV samples were obtained from patients with metastasis. The indication for surgery was according to tumor size, age and patient aspirations. The clinical information of patients are summarized in Table I. Pertinent follow-up information was available for all patients. The cut-off value was decided by the ROC curve. Written informed consent was obtained from each patient and established study approval was obtained from the Institutional Research Ethics Committee from Southern Medical University.
Table I. Clinicopathologic variables in 55 patients with hepatocellular carcinoma.

\begin{tabular}{|c|c|c|c|c|}
\hline \multirow[b]{2}{*}{ Variable } & \multirow[b]{2}{*}{$\mathrm{n}$} & \multicolumn{2}{|c|}{ SIRT5 protein expression } & \multirow[b]{2}{*}{ P-value } \\
\hline & & Low $(n=20)$ & $\operatorname{High}(\mathrm{n}=35)$ & \\
\hline \multicolumn{5}{|l|}{ Age (years) } \\
\hline$<50$ & 32 & 12 & 20 & 0.836 \\
\hline$\geq 50$ & 23 & 8 & 15 & \\
\hline \multicolumn{5}{|l|}{ Sex } \\
\hline Male & 38 & 14 & 24 & 0.912 \\
\hline Female & 17 & 6 & 11 & \\
\hline \multicolumn{5}{|l|}{ Tumor size } \\
\hline Small $(\leq 5 \mathrm{~cm})$ & 20 & 11 & 9 & 0.030 \\
\hline Large $(\geq 5 \mathrm{~cm})$ & 35 & 9 & 26 & \\
\hline \multicolumn{5}{|l|}{$\begin{array}{l}\text { Lymph node } \\
\text { metastasis }\end{array}$} \\
\hline Yes & 27 & 5 & 22 & 0.007 \\
\hline No & 28 & 15 & 13 & \\
\hline \multicolumn{5}{|l|}{ TNM stage } \\
\hline $\mathrm{I}+\mathrm{II}$ & 27 & 14 & 13 & 0.019 \\
\hline III+IV & 28 & 6 & 22 & \\
\hline \multicolumn{5}{|l|}{ Differentiation } \\
\hline Well/moderate & 29 & 11 & 18 & 0.799 \\
\hline Poor & 26 & 9 & 17 & \\
\hline
\end{tabular}

SIRT5, sirtuin 5; TNM, tumor-node-metastasis.

RNA extraction and RT-qPCR analysis. Total RNA was extracted from the frozen human clinical tissues and cultured cell lines using TRIzol reagent (Invitrogen Life Technologies; Thermo Fisher Scientific, Inc.) according to the manufacturer's protocol. The RNA (2 $\mu \mathrm{g})$ was used for first strand cDNA synthesis using the Revert Aid ${ }^{\mathrm{TM}}$ First Strand cDNA Synthesis kit (Fermentas; Thermo Fisher Scientific, Inc.). In order to quantify the transcripts of the genes, qPCR was performed using a SYBR-Green mixture $2 \mu \mathrm{l}$ cDNA, $1 \mu \mathrm{l}$ primers, $10 \mu \mathrm{l}$ mixture buffer and $7 \mu \mathrm{l}$ water (Qiagen, Inc., Valencia, CA, USA) on an ABI 7500 system (Applied Biosystems; Thermo Fisher Scientific, Inc.). The primers used were as follows: GAPDH forward, 5'-GAGAAGTATGACAACAGCCTC-3' and reverse, 5'-ATGGACTGTGGTCATGAGTC-3'; SIRT5 forward, 5'-AAATAACTAAAGCCCGCCTC-3' and reverse, 5'-TCCTGAGATGATGACTATGTG-3'; and E2F1 forward, 5'-GACTCTTCGGAGAACTTTCAG-3' and reverse, 5'-GAT CTGTGGTGAGGGATGAG-3'. The PCR amplification was $95^{\circ} \mathrm{C}$ for $5 \mathrm{~min}$, followed by 35 cycles of $95^{\circ} \mathrm{C}$ for $20 \mathrm{sec}, 60^{\circ} \mathrm{C}$ for $15 \mathrm{sec}$, and $72^{\circ} \mathrm{C}$ for $30 \mathrm{sec}$. The genes expression were normalized against that of GAPDH and relative fold changes were calculated using the formula $2^{-\Delta \Delta \mathrm{Cq}}(11)$.

Western blot analysis. The relative tissues or cells were lysed in $50 \mathrm{mM}$ Tris $\mathrm{HCl}$ (pH 7.5; Beyotime Institute of Biotechnology, Shanghai, China) and 1\% SDS with protease/phosphatase inhibitor cocktail (Roche Diagnostics, Basel, Switzerland), 
and then heated at $95^{\circ} \mathrm{C}$ for $10 \mathrm{~min}$. The protein was quantified using a BCA kit (Thermo Fisher Scientific, Inc.). Equal quantities of protein $(30 \mu \mathrm{g})$ were subjected to $10 \%$ SDS-PAGE, and transferred onto polyvinylidene fluoride membranes (EMD Millipore, Billerica, MA, USA). Following blocking in 5\% nonfat milk with PBS containing 0.1\% Tween-20, the membranes were incubated with primary antibodies anti-SIRT5 antibody (1:1,000, cat. no. ab105040), anti-E2F1 antibody $(1: 1,000$, cat. no. ab179445) and anti- $\beta$-actin antibody $(1: 1,000$, cat. no. ab8226) at $4^{\circ} \mathrm{C}$ overnight, and then washed for least five times. This was followed by incubation with horseradish peroxidase-labeled secondary antibodies (Santa Cruz Biotechnology, Inc.) goat anti-mouse IgG-horseradish peroxidase (sc-2005; 1:3,000) and anti-rabbit IgG-horseradish peroxidase (sc-2004; $1: 3,000)$ at room temperature for $1 \mathrm{~h}$. The membranes were detected using an enhanced chemiluminescence-based method.

Quantitative chromatin immunoprecipitation (qChIP) analysis. The qChIP assay was performed using the Chip-IT Express kit (Active Motif, Carlsbad, CA, USA). The relative cultured cells were cross-linked with formaldehyde, incubated with ChIP lysis buffer for $30 \mathrm{~min}$, and sonicated to generate 200-500-bp DNA fragments at $4^{\circ} \mathrm{C}$. Supernatant was incubated with $5 \mu \mathrm{g}$ relative antibody, or normal $\operatorname{IgG}$ as a negative control, overnight at $4^{\circ} \mathrm{C}$. Following being washed five times with lysis buffer, the DNA was de-crosslinked, extracted using phenol chloroform and precipitated with ethanol. Following resuspension in $50 \mu \mathrm{l}$ of $\mathrm{H}_{2} \mathrm{O}$, qPCR was performed with $5 \mu 1$ of the immunoprecipitated target DNA, $1 \mu \mathrm{l}$ primers and $9 \mu \mathrm{l}$ mixture $(1 \mu \mathrm{l}$ enzyme, $2 \mu \mathrm{l}$ dNTP and $6 \mu 1$ solution buffer). The qChIP primers were as follows: E2F1 forward, 5'-CTGGGTGCAGGATTGGATA-3' and reverse, 5'-TCAACCTGTAGCCCCCAAC-3'. The PCR amplification was performed at $95^{\circ} \mathrm{C}$ for $5 \mathrm{~min}$, followed by 35 cycles of $95^{\circ} \mathrm{C}$ for $20 \mathrm{sec}, 55^{\circ} \mathrm{C}$ for $20 \mathrm{sec}$, and $72^{\circ} \mathrm{C}$ for $30 \mathrm{sec}$. Bioinformatics was performed using PROMO (http://alggen.lsi.upc. es/cgi-bin/promo_v3/promo/promoinit.cgi?dirDB=TF_8.3) to submit the promoter sequence of E2F1 to find the potential binding of SIRT5.

Luciferase reporter gene assay. A luciferase reporter gene assay was performed to validate the predicted target. Briefly, the promoter region of E2F1 containing the putative binding site was cloned into a PGL3 luciferase reporter vector (Promega, Madison, WI, USA). The HCC cells $\left(1 \times 10^{3}\right)$ were seeded in 96-well plates, and the E2F1 reporter plasmid was cotransfected into cells with $2 \mathrm{ng} /$ well Renilla luciferase construct in triplicate. After 36 h, the activities of Firefly and Renilla luciferase were determined using a dual luciferase detection kit (Promega).

Proliferation assay. A Cell Counting kit-8 (CCK-8; Dojindo Molecular Technologies, Inc., Kumamoto, Japan) was used to detect cell proliferation. The relative transfected cells $\left(5 \times 10^{3}\right.$ cells) were plated on 48 -well plates and cultured overnight to allow attachment. Following serum-starvation for $8 \mathrm{~h}$, FBS was added into the medium. At 0, 12, 24, 36, 48 and $72 \mathrm{~h}$, the cells were incubated with $10 \mu \mathrm{l}$ CCK-8 solution for $2 \mathrm{~h}$, and analyzed at $450 \mathrm{~nm}$ using a multi-well plate reader. The experiments were performed in triplicate.
Invasion assay. An invasion assay was performed using Transwell chambers with inserts of 8-mm pore size (Corning Costar; Corning Inc., Corning, NY, USA). Briefly, the relative hepatoma cells $\left(1 \times 10^{5}\right)$ were suspended in serum-free medium and seeded into Matrigel invasion chambers at $37^{\circ} \mathrm{C}$. Complete medium (DMEM with 10\% FBS) was added to the lower chamber. Following cultured for $72 \mathrm{~h}$, the cells on the lower surface were stained with crystal violet. The cell numbers were counted under a microscope (magnification, $\mathrm{x} 40$ ) (Leica DM4B; Leica Microsystems, Inc., Buffalo Grove, IL, USA) in five randomly selected fields in each well.

Statistical analysis. Statistical analysis was performed using SPSS version 17.0 (SPSS, Inc., Chicago, IL, USA). Data are expressed as the mean \pm standard deviation of three independent experiments. Student's t-test or one-way analysis of variance were used, as appropriate. A Kaplan-Meier plot was used to analyze the survival curves and a log-rank test was used to compare the survival curves. The association between expression levels and clinicopathological characteristics were analyzed using a $\chi^{2}$ test and Spearman's correlation. $\mathrm{P}<0.05$ was considered to indicate a statistically significant difference.

\section{Results}

SIRT5 is upregulated in HCC cell lines and tumor tissues. RT-qPCR analysis was used to analyze the expression of SIRT5 in 55 surgical HCC specimens. As shown in Fig. 1A, the levels of SIRT5 were significantly upregulated in the majority of the HCC tissues, compared with those in the paired adjacent noncancerous tissues. The upregulation of SIRT5 in HCC tissues suggested that SIRT5 may be important in the progression of HCC. The expression of SIRT5 was further examined in 20 metastatic and primary HCC tissues. As shown in Fig. 1B, there was a significantly higher expression of SIRT5 in the metastatic tissues, compared with the primary HCC samples. In subsequent experiments, RT-qPCR analysis was performed to detect the expression of SIRT5 in different HCC cell lines (SMMC-7721, HepG2, LO2 and Huh7 cells). It was found that the expression of SIRT5 was generally higher in these HCC cell lines, compared with that in the non-tumorigenic immortalized liver cell line (MiHA), as shown in Fig. 1C.

High expression of SIRT5 is associated with a poor clinical prognosis in HCC. To further elucidate the clinical relevance of the expression of SIRT5 in HCC, the present study investigated the association between the expression of SIRT5 and clinicopathological features. As summarized in Table I, the higher expression level of SIRT5 in HCC was positively correlated with tumor size, lymph node metastasis status and TNM stage. However, no significant correlations were observed between the expression levels of SIRT5 and the clinical features of sex, age or degree of tumor differentiation. These data indicated that SIRT5 may be involved in the progression of HCC. Kaplan-Meier analysis was also performed to analyze the association between tumor expression and patient overall survival rates. As shown in Fig. 2, patients with a high expression of SIRT5 had a poorer OS, compared with patients with a low expression of SIRT5 (log-rank test, $\mathrm{P}<0.001$ ). 
A

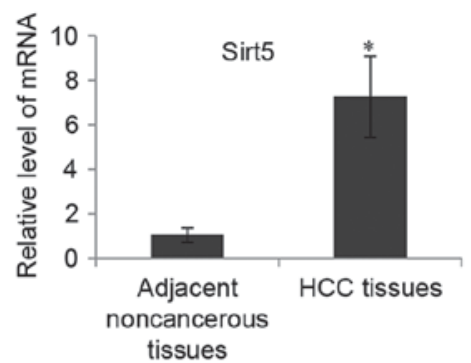

B

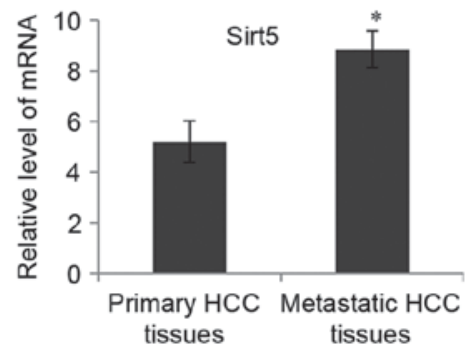

C

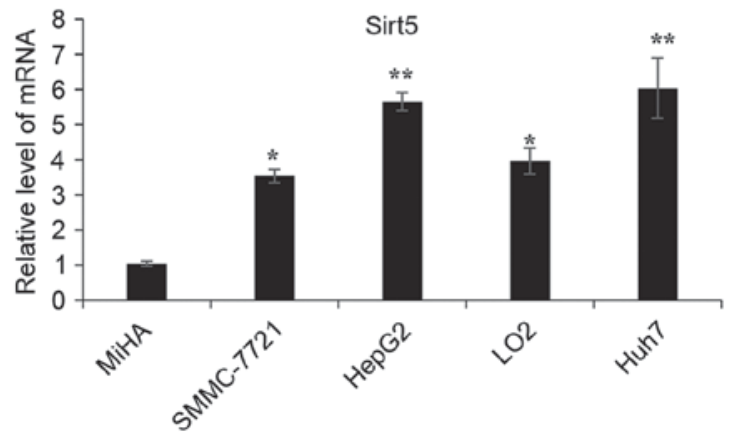

Figure 1. SIRT5 is upregulated in HCC cell lines and tumor tissues (A) RT-qPCR analysis of the expression levels of SIRT5 in 55 HCC tissues and paired non-tumor tissues. (B) RT-qPCR analysis of the expression levels of SIRT5 in 20 metastatic and paired primary HCC tissues. (C) mRNA levels of SIRT5 were determined by RT-qPCR analysis in HCC cell lines and MiHA cells. Three independent experiments were performed. Data are presented as the mean \pm standard deviation. ${ }^{*} \mathrm{P}<0.05 ;{ }^{* *} \mathrm{P}<0.01$. SIRT5, sirtuin 5; HCC, hepatocellular carcinoma; RT-qPCR, reverse transcription-quantitative polymerase chain reaction analysis.

SIRT5 promotes HCC cell growth in vitro. The high expression levels of SIRT5 in HCC suggested that SIRT5 may be involved in the development and prognosis of HCC. To determine whether SIRT5 is essential for HCC cell growth, two types of lentiviral vector carrying various sh-RNA sequences to knockdown SIRT5 (shSIRT5) were stably expressed in Huh7 and HepG2 cells. As shown in Fig. 3A and B, shSIRT5-1 and shSIRT5-2 were able to efficiently reduce the expression of SIRT5 at the mRNA level. Cell proliferation was detected using a CCK-8 assay in the two cell lines, and the cells stably expressing shSIRT5-1 or shSIRT5-2 showed significantly decreased cell proliferation, compared with the control (Fig. 3C and D).

SIRT5 regulates HCC cell invasion in vitro. To better understand the functionality of SIRT5 in the progression of HCC, the present study examined whether SIRT5 can exert an effect on HCC cell invasion. A Transwell assay was performed and, as expected, the depletion of SIRT5 in Huh7 cells resulted in a marked decrease in cell invasion, as shown in the images in Fig. 4A and the statistical analyses in Fig. 4B. A Transwell

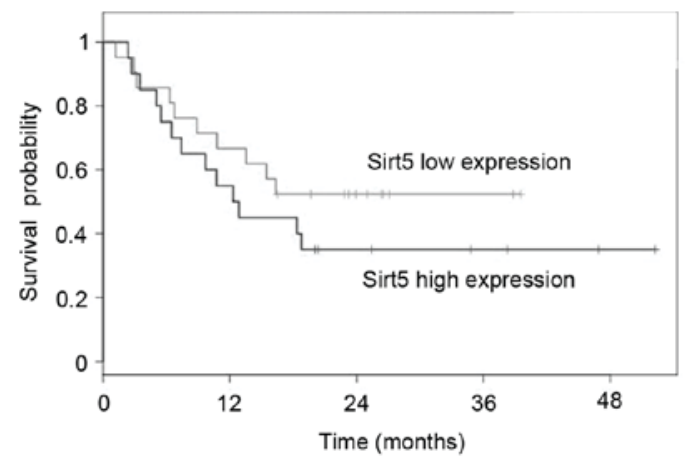

Figure 2. High expression of SIRT5 is associated with poor clinical prognosis in hepatocellular carcinoma. Kaplan-Meier plot of OS using the log-rank test. In patients with high levels of SIRT5, OS was significantly lower, compared with that in patients with low levels of SIRT5. OS, overall survival; SIRT5, sirtuin 5.

assay was also performed for the HepG2 cells, and a similar trend was observed (Fig. 4C).

SIRT5 directly targets E2F1 and activates its expression. Previous studies $(12,13)$ have shown that sirtuins can regulate the expression of several genes at the transcriptional or post-transcriptional level. To identify potential target genes of SIRT5, bioinformatics analysis was used, which revealed that SIRT5 directly targeted E2F1, an important regulator involved in tumor progression. To further examine this, a qChIP assay was performed in the huh7 cells and HepG2 cells (Fig. 5A). Compared with the normal $\mathrm{IgG}$ group, there was binding enrichment of SIRT5 on the promoter of E2F1. A luciferase reporter vector containing the promoter of $\mathrm{E} 2 \mathrm{~F} 1$ was also constructed, and the reporter assay showed that the knockdown of SIRT5 was able to significantly reduce the expression of E2F1 luciferase in the two cell lines (Fig. 5B). Consistently, shSIRT5 significantly inhibited the mRNA levels and inhibited protein levels of E2F1 in the Huh7 and HepG2 cells (Fig. 5C and D). These data demonstrated that SIRT5 directly targeted E2F1 and activated the endogenous expression of E2F1 in HCC cells.

SIRT5 promotes HCC cell proliferation and invasion by targeting E2F1. The above results showed that SIRT5 acted primarily as a promoter of HCC cell proliferation and invasion, and identified E2F1 as a target gene. To evaluate the direct effect of E2F1 in the growth and invasive potential of SIRT5, two shRNAs (shE2F1-1 and shE2F1-2) targeting E2F1 were used to suppress its expression. As shown in Fig. 6A and B, the shRNAs efficiently reduced the expression of E2F1 at the mRNA level, compared with the NC group and mock-treated group. As shown in Fig. 6C and D, the knockdown of SIRT5 inhibited Huh7 cell and HepG2 cell growth, and the additional knockdown of E2F1 in the two cells partially reversed the inhibitory effect of SIRT5-knockdown. Similarly, SIRT5 promoted Huh7 or HepG2 cell invasion by regulating the expression of E2F1, as the knockdown of E2F1 partially reduced the effect of SIRT5 (Fig. 6E and F).

\section{Discussion}

In previous years, the high recurrence rate of $\mathrm{HCC}$ has remained a major challenge in improving the outcome for 


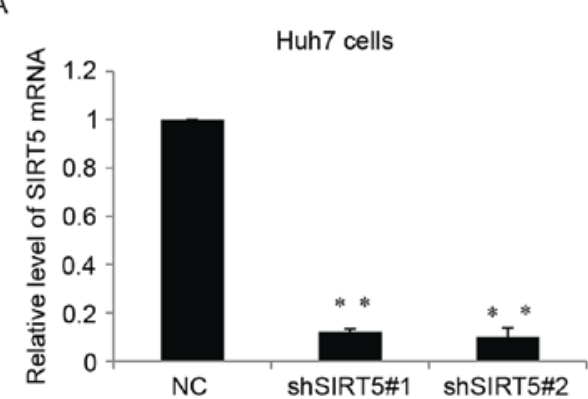

C

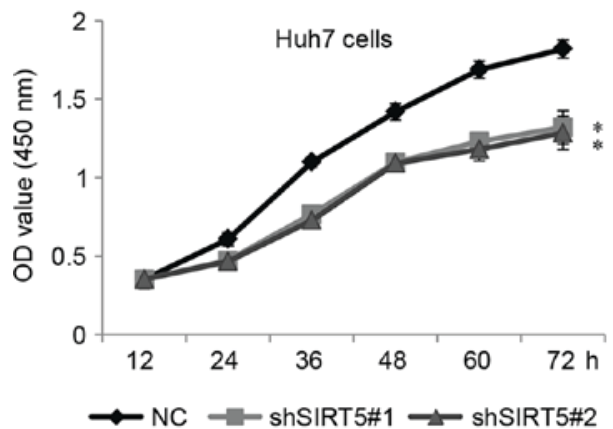

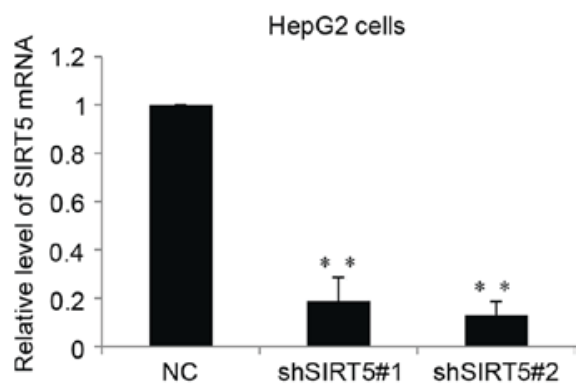

D

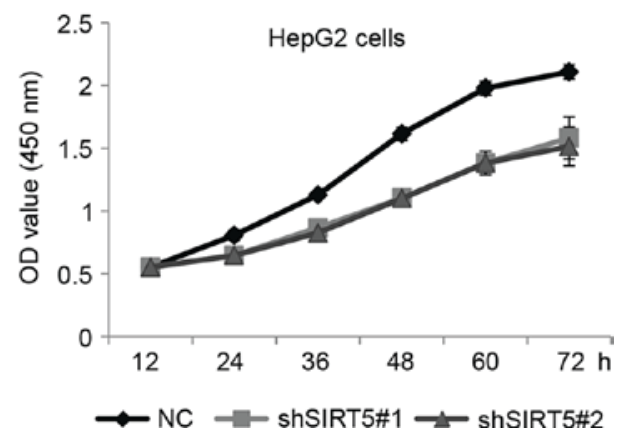

Figure 3. SIRT5 promotes hepatocellular carcinoma cell growth in vitro. (A) mRNA levels of SIRT5 were measured in Huh7 cells stably transfected with shSIRT5\#1, shSIRT5\#2 or NC. Data are presented as the mean \pm standard deviation. ${ }^{* *} \mathrm{P}<0.01$. (B) Relative mRNA levels of SIRT5 in HepG2 cells. (C) CCK-8 assay of Huh7 cells, which were stably transfected with either shSIRT5\#1, shSIRT5\#2 or NC, at 0, 12, 24, 36,48 and 72 h. ** P<0.01. (D) Relative CCK-8 assay in HepG2 cells. SIRT5, sirtuin 5; sh, short hairpin RNA; NC, negative control; CCK-8, Cell Counting kit-8; OD, optical density.

A

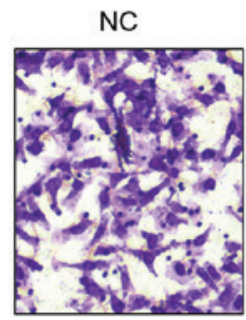

shSIRT5\#1

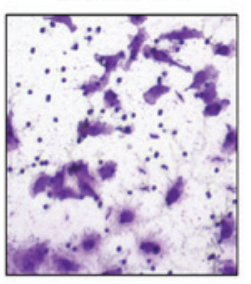

ShSIRT5\#2

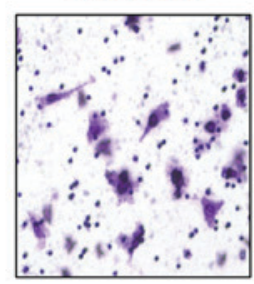

B

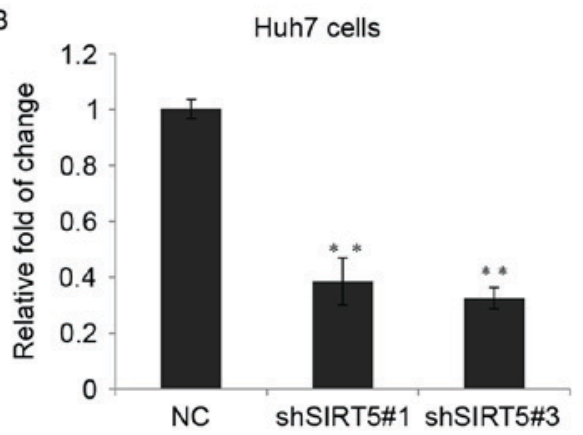

C

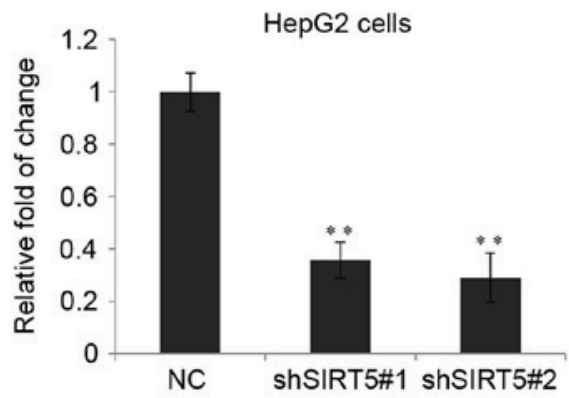

Figure 4. SIRT5 regulates hepatocellular carcinoma cell invasion in vitro. (A) Huh7 cells were transfected with NC, shSIRT5\#1 or shSIRT5\#2, and cell invasion was determined using a Transwell assay. Representative images (magnification, x20) are shown. (B) Transwell assays were performed in triplicate and data are expressed as the mean \pm standard deviation. ${ }^{* *} \mathrm{P}<0.01$ vs. $\mathrm{NC}$ group. (C) Transwell assays were performed in HepG2 cells in triplicate, with data expressed as the mean \pm standard deviation. ${ }^{* *} \mathrm{P}<0.01$ vs. NC group. SIRT5, sirtuin 5; sh, short hairpin RNA; NC, negative control.

patients with HCC. Therefore, identifying early therapeutic targets is important for improved clinical outcome. In the present study, it was shown that SIRT5 was overexpressed in the majority of primary HCC tumor tissues, compared with corresponding non-tumorous liver tissues. The data revealed that the expression of SIRT5 was significantly upregulated in HCC tissues, and that higher expression levels of SIRT5 were observed in metastatic HCC samples. The expression pattern of SIRT5 found in the present study was consistent with previous investigations, in which SIRT5 was also overexpressed in 
A

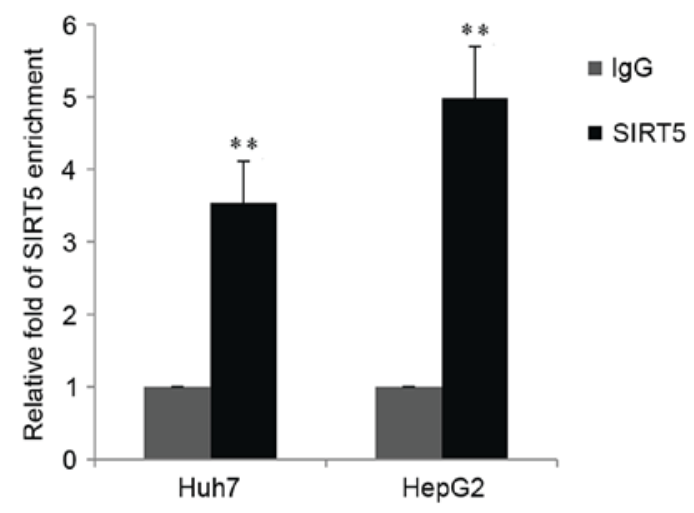

B

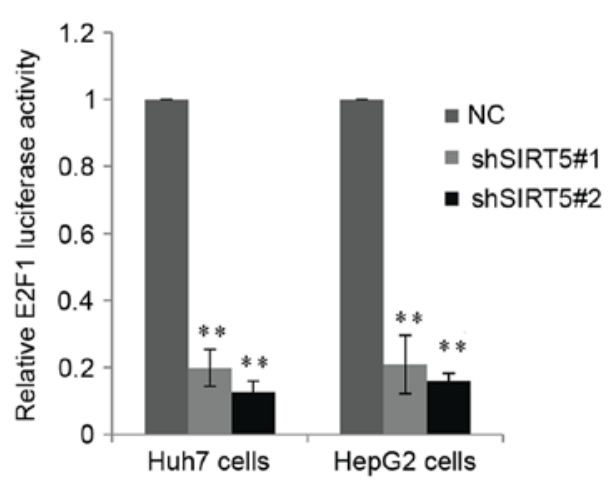

D

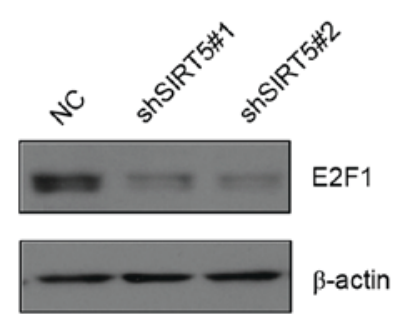

C

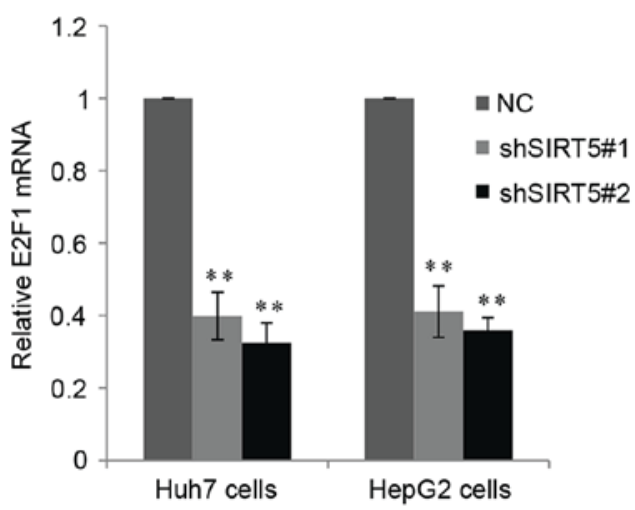

E

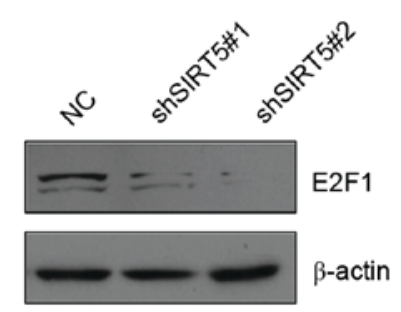

Figure 5. SIRT5 directly targets E2F1 and activates its expression. (A) Quantitative chromatin immunoprecipitation assay in Huh7 cells and HepG2 cells. (B) Luciferase reporter assays were performed in Huh7 cells and HepG2 cells transfected with pGL3-E2F1, co-transfected with Renilla and either NC, shSIRT5\#1 or shSIRT5\#2. Firefly luciferase reporter activity was determined $48 \mathrm{~h}$ following transfection and standardized to the Renilla control. (C) RT-qPCR analysis of the endogenous mRNA levels of E2F1 following transfection with NC, shSIRT5\#1 or shSIRT5\#2. Data are presented as the mean \pm standard deviation of triplicate experiments. ${ }^{* *} \mathrm{P}<0.01$. (D) Western blot analysis for endogenous protein levels of E2F1 following SIRT5 inhibition in hepatoma Huh7 cells. (E) Western blot analysis for endogenous protein levels of E2F1 following SIRT5 inhibition in hepatoma HepG2 cells. SIRT5, sirtuin 5; E2F1, E2F transcription factor 1; sh, short hairpin RNA; NC, negative control; RT-qPCR, reverse transcription-quantitative polymerase chain reaction.

human non-small cell lung cancer, with a high expression of SIRT5 predictive of poor survival rates $(13,14)$. Further statistical analysis found that a higher expression of SIRT5 in the HCC tissues was significantly associated with malignant tumor characteristics, including tumor size, lymph node metastasis and TNM stage. Therefore, these results demonstrated that SIRT5 is a potential promoter of metastasis in HCC. Kaplan-Meier survival analysis also revealed that the higher expression of SIRT5 was associated with poor prognosis following surgical resection in patients with $\mathrm{HCC}$.

The above findings suggested that SIRT5 may serve as a novel prognostic predictor and therapeutic target in patients with HCC. By obtaining in vitro evidence using HCC cell lines, the present study demonstrated that SIRT5 facilitated cell growth and invasion of the selected HCC cell lines. Mechanistically, it was observed that SIRT5 regulated the expression of E2F1.
As a founding member of the E2F transcription factors, E2F1 is involved in regulating cell differentiation, DNA repair and apoptosis $(15,16)$. E2F1 also functions as a connector and coordinator between cell proliferation and metabolic pathways in mitochondria. Mitochondria are multifunctional organelles, which are suggested to be actively involved in various aspects of tumorigenesis (17). It has been reported that E2F1 enhances glycolysis through suppressing the transcription of Sirt6 in PC3 prostate cancer and UMUC3 bladder cancer cells (18). In vitro studies have shown that E2F1 may act either as an oncogene or as a tumor suppressor (19). E2F1 is overexpressed and pro-apoptotic in human HCC (20) and acts as a growth-promoting factor in non-small cell lung carcinoma, which is associated with adverse prognosis, and as a promoter of invasion and metastasis $(21,22)$. The present study revealed that, through the activation of E2F1, SIRT5 promotes HCC proliferation and invasion. However, the present study was 
A

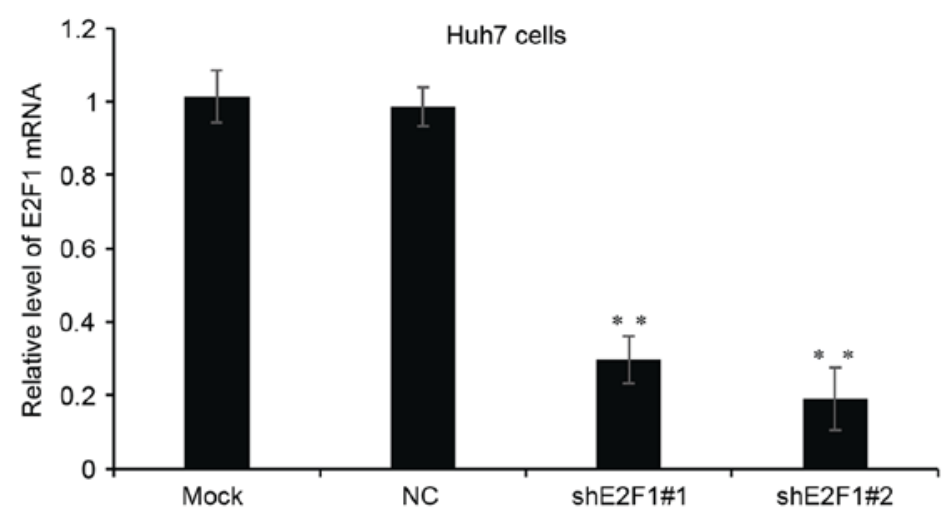

B

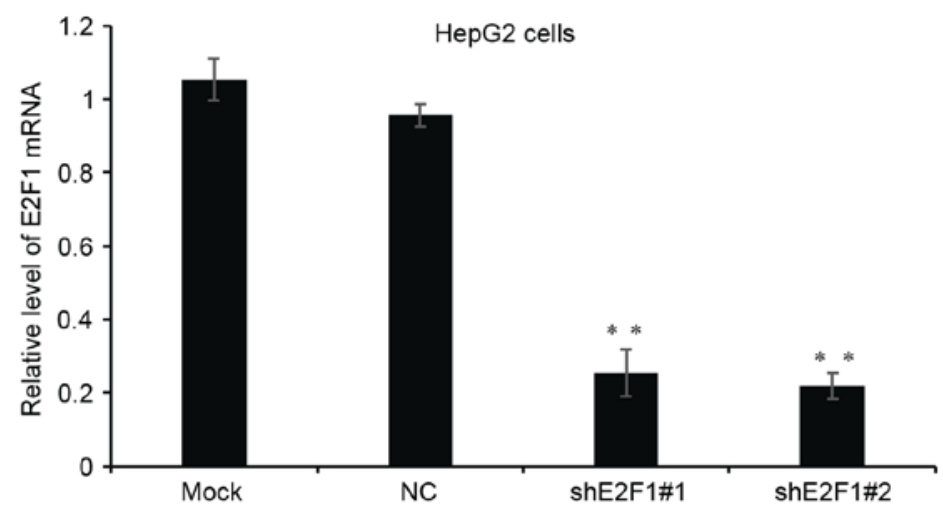

C

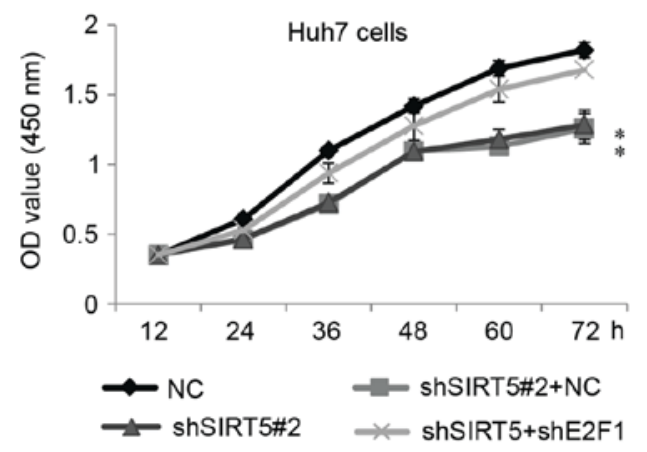

E

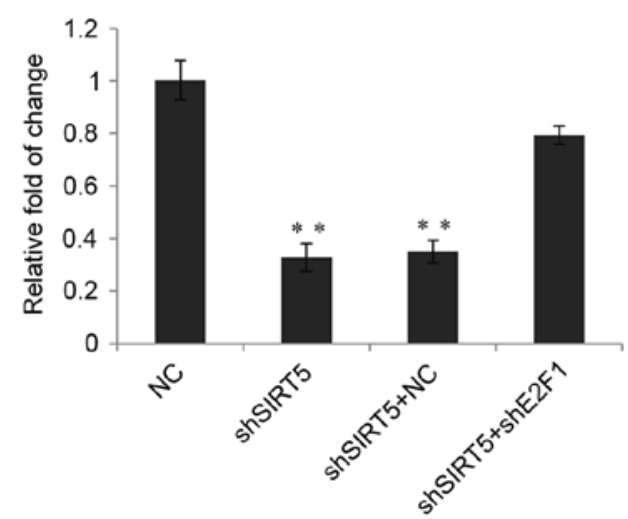

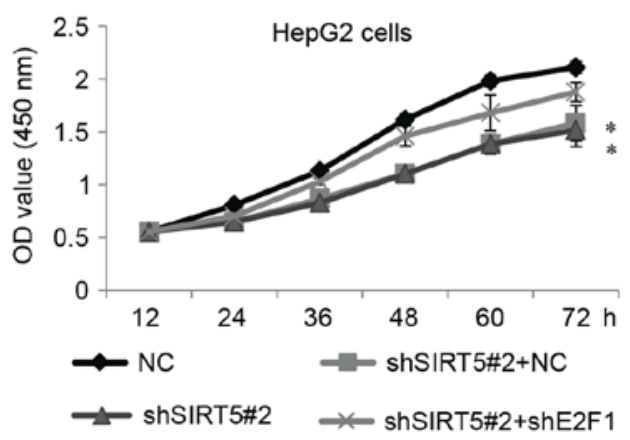

F

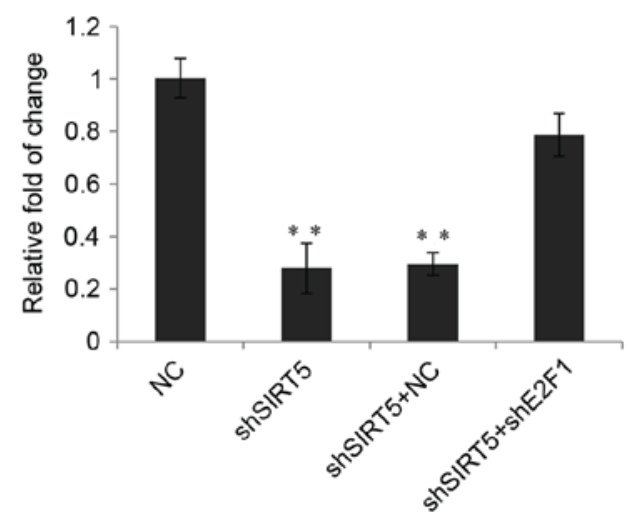

Figure 6. SIRT5 promotes hepatocellular carcinoma cell proliferation and invasion by targeting E2F1. (A) mRNA levels of E2F1 were measured in Huh7 cells stably transfected with shE2F1\#1, shE2F1\#2, NC and in untreated (Mock) cells. Data are presented as the mean \pm standard deviation. ${ }^{* *} \mathrm{P}<0.01$. (B) Relative mRNA levels of E2F1 were measured in HepG2 cells. (C) CCK-8 assay of Huh7 cells stably transfected with either NC, shSIRT5+NC or shSIRT5+shE2F1 at 0, 12, 24, 36, 48 and $72 \mathrm{~h} .{ }^{* *} \mathrm{P}<0.01$. (D) Relative CCK-8 assay was performed in HepG2 cells. (E) Cell invasion assay was performed in Huh7 cells transfected with NC, shSIRT5, shSIRT5+NC or shSIRT5+shE2F1. Data are presented as the mean \pm standard deviation of at least three independent experiments. (F) Relative Transwell assay in HepG2 cells. SIRT5, sirtuin 5; E2F1, E2F transcription factor 1; sh, short hairpin RNA; NC, negative control; OD, optical density; CCK-8, Cell Counting kit-8. 
limited by the low quantity of patient tissue samples, as there was insufficient tissue to measure protein expression levels to determine their association with SIRT5 and E2F1.

As the most well-characterized member of the sirtuin family, it has been reported that, in HCC, the overexpression of SIRT1 can promote metastasis through epithelial-mesenchymal transition (EMT) (23). This suggests that SIRT5 may also regulate HCC cell invasion through the EMT progress, which is reported to be associated with liver cancer migration and metastasis $(24,25)$ EMT-associated transcription factors may be target genes of SIRT1 in HCC, therefore, additional target genes require investigation in the future.

\section{References}

1. Jemal A, Bray F, Center MM, Ferlay J, Ward E and Forman D: Global cancer statistics. CA Cancer J Clin 61: 69-90, 2011.

2. Forner A, Llovet JM and Bruix J: Hepatocellular carcinoma. Lancet 379: 1245-1255, 2012.

3. Bosch FX, Ribes J, Díaz M and Cléries R: Primary liver cancer: Worldwide incidence and trends. Gastroenterology 127 (5 Suppl 1): S5-S16, 2004.

4. Alazawi W, Cunningham M, Dearden J and Foster GR: Systematic review: Outcome of compensated cirrhosis due to chronic hepatitis C infection. Aliment Pharmacol Ther 32: 344-355, 2010.

5. Bertino G, Demma S, Ardiri A, Proiti M, Gruttadauria S, Toro A, Malaguarnera G, Bertino N, Malaguarnera M, Malaguarnera M and Di Carlo I: Hepatocellular carcinoma: Novel molecular targets in carcinogenesis for future therapies. Biomed Res Int 2014: 203693, 2014.

6. Faivre S, Bouattour M and Raymond E: Novel molecular therapies in hepatocellular carcinoma. Liver Int 31 (Suppl 1): S151-S160, 2011

7. Liang XJ, Finkel T, Shen DW, Yin JJ, Aszalos A and Gottesman MM: SIRT1 contributes in part to cisplatin resistance in cancer cells by altering mitochondrial metabolism. Mol Cancer Res 6: 1499-1506, 2008 .

8. Michan S and Sinclair D: Sirtuins in mammals: Insights into their biological function. Biochem J 404: 1-13, 2007.

9. Dali-Youcef N, Lagouge M, Froelich S, Koehl C, Schoonjans K and Auwerx J: Sirtuins: The 'magnificent seven', function, metabolism and longevity. Ann Med 39: 335-345, 2007.

10. Varotti G, Ramacciato G, Ercolani G, Grazi GL, Vetrone G, Cescon M, Del Gaudio M, Ravaioli M, Ziparo V, Lauro A and Pinna A: Comparison between the fifth and sixth editions of the AJCC/UICC TNM staging systems for hepatocellular carcinoma: Multicentric study on 393 cirrhotic resected patients. Eur J Surg Oncol 31: 760-767, 2005.
11. Livak KJ and Schmittgen TD: Analysis of relative gene expression data using real-time quantitative PCR and the 2(-Delta Delta C(T)) method. Methods 25: 402-408, 2001.

12. Carafa V, Rotili D, Forgione M, Cuomo F, Serretiello E, Hailu GS, Jarho E, Lahtela-Kakkonen M, Mai A and Altucci L: Sirtuin functions and modulation: From chemistry to the clinic. Clin Epigenetics 8: 61, 2016.

13. Kida $\mathrm{Y}$ and Goligorsky MS: Sirtuins, cell senescence and vascular aging. Can J Cardiol 32: 634-641, 2016.

14. Lu W, Zuo Y, Feng Y and Zhang M: SIRT5 facilitates cancer cell growth and drug resistance in non-small cell lung cancer. Tumour Biol 35: 10699-10705, 2014.

15. Iaquinta PJ and Lees JA: Life and death decisions by the E2F transcription factors. Curr Opin Cell Biol 19: 649-657, 2007.

16. Polager S and Ginsberg D: E2F - at the crossroads of life and death. Trends Cell Biol 18: 528-535, 2008.

17. Mori K, Uchida T, Fukumura M, Tamiya S, Higurashi M, Sakai H, Ishikawa F and Shibanuma M: Linkage of E2F1 transcriptional network and cell proliferation with respiratory chain activity in breast cancer cells. Cancer Sci 107: 963-971, 2016.

18. Wu M, Seto E and Zhang J: E2F1 enhances glycolysis through suppressing Sirt6 transcription in cancer cells. Oncotarget 6: $11252-11263,2015$

19. Tsantoulis PK and Gorgoulis VG: Involvement of E2F transcription factor family in cancer. Eur J Cancer 41: 2403-2414, 2005.

20. Palaiologou M, Koskinas J, Karanikolas M, Fatourou E and Tiniakos DG: E2F-1 is overexpressed and pro-apoptotic in human hepatocellular carcinoma. Virchows Arch 460: 439-446, 2012.

21. Gorgoulis VG, Zacharatos P, Mariatos G, Kotsinas A, Bouda M, Kletsas D, Asimacopoulos PJ, Agnantis N, Kittas C and Papavassiliou AG: Transcription factor E2F-1 acts as a growth-promoting factor and is associated with adverse prognosis in non-small cell lung carcinomas. J Pathol 198: 142-156, 2002.

22. Gu Y, Cheng Y, Song Y, Zhang Z, Deng M, Wang C, Zheng G and He Z: MicroRNA-493 suppresses tumor growth, invasion and metastasis of lung cancer by regulating E2F1. PLoS One 9: e102602, 2014

23. Hao C, Zhu PX, Yang X, Han ZP, Jiang JH, Zong C, Zhang XG, Liu WT, Zhao QD, Fan TT, et al: Overexpression of SIRT1 promotes metastasis through epithelial-mesenchymal transition in hepatocellular carcinoma. BMC Cancer 14: 978, 2014.

24. Mima K, Hayashi H, Kuroki H, Nakagawa S, Okabe H, Chikamoto A, Watanabe M, Beppu T and Baba H: Epithelialmesenchymal transition expression profiles as a prognostic factor for disease-free survival in hepatocellular carcinoma: Clinical significance of transforming growth factor- $\beta$ signaling. Oncol Lett 5: 149-154, 2013.

25. Li YM, Xu SC, Li J, Han KQ, Pi HF, Zheng L, Zuo GH, Huang XB, Li HY, Zhao HZ, et al: Epithelial-mesenchymal transition markers expressed in circulating tumor cells in hepatocellular carcinoma patients with different stages of disease. Cell Death Dis 4: e831, 2013. 